\title{
The most influential articles on kidney transplantation: a bibliometric and visualized analysis
}

Heungman Jun

Department of Surgery-Transplantation, Inje University Ilsan Paik Hospital, Goyang, Korea

Background: Kidney transplantation has become common in the treatment of end-stage renal disease. However, to date, there has been no bibliometric analysis of kidney transplantation research to identify the most influential articles. The purpose of this research was to identify and characterize the 100 most cited articles that focused on kidney transplantation and to clarify the trends in the accomplishments in this field.

Methods: We searched the Thomson Reuters Web of Science citation indexing database and used keyword mapping of VOSviewer. The top 100 most cited manuscripts were analyzed based on the title, author, institution, country of origin, year of publication, and topic.

Results: The New England Journal of Medicine published the most manuscripts on kidney transplantation ( $\mathrm{n}=26)$ and was the most cited journal $(n=15,642)$. The United States had the highest number of publications $(n=61)$. Kashika was the corresponding author with the most published papers ( $n=5 ; 2,892$ citations). The most common topics of publication were immunosuppressant $(n=34)$, clinical outcome $(n=26)$, and pathology $(n=22)$. Keywords related to immunosuppressant were the most common in keyword mapping with VOSviewer.

Conclusions: This bibliometric analysis of kidney transplantation research provides the research characteristics and publication trends.

Corresponding author: Heungman Jun

E-mail: midasia@hanmail.net

(c) The Korean Society for Transplantation

This is an Open Access article distributed under the terms of the Creative Commons Attribution Non-Commercial License (http://creativecommons.org/licenses/by-nc/4.0/) which permits unrestricted non-commercial use, distribution, and reproduction in any medium, provided the original work is properly cited. 